\title{
EFEITOS DA OMISSÃO DE NUTRIENTES EM PLANTAS DE Caesalpinia echinata
}

\author{
Sergio Valiengo Valeri ${ }^{1 *}$, Luiz Gustavo Ennes Pizzaia ${ }^{1}$, Ariadne Felício Lopo de Sá1, Mara Cristina Pessoa da Cruz ${ }^{1}$ \\ *Autor para correspondência: valeri@fcav.unesp.br
}

\begin{abstract}
RESUMO: O objetivo deste trabalho foi avaliar o crescimento, as alterações morfológicas e a composição mineral de plantas de pau-brasil (Caesalpinia echinata) decorrentes da omissão de nutrientes pela técnica do elemento faltante em experimento em casa de vegetação. O delineamento experimental foi inteiramente casualizado, com 12 tratamentos: testemunha (solo natural), completo (N, $\mathrm{P}, \mathrm{K}, \mathrm{Ca}, \mathrm{Mg}, \mathrm{S}, \mathrm{B}, \mathrm{Cu}, \mathrm{Mn}$ e $\mathrm{Zn}$ ), e adubação com omissão de cada um dos nutrientes do tratamento completo e cinco repetições. Cada parcela foi constituída por uma planta em vaso contendo $7 \mathrm{dm}^{3}$ de Neossolo Quartzarênico. Foram avaliados sintomas visuais de deficiência nutricional, altura, diâmetro do caule, matéria seca da parte aérea das plantas, incluindo caule, ramos e folhas e teor dos nutrientes nas folhas. A omissão de nitrogênio em Neossolo Quartzarênico limitou o crescimento em altura e a produção de biomassa da parte aérea das plantas de pau-brasil. Os primeiros sintomas de deficiência nutricional foram decorrentes da omissão de $\mathrm{N}$, em seguida apareceram os sintomas de $\mathrm{P}, \mathrm{Ca}, \mathrm{Mg}, \mathrm{S}, \mathrm{Cu}$ e $\mathrm{Mn}$ e, mais tardiamente, os sintomas da deficiência de K e B. Para cada nutriente, houve diferença entre as médias de teores nas folhas em função dos tratamentos.
\end{abstract}

Palavras chave: pau-brasil, deficiência nutricional, diagnose, nutrição florestal.

\section{EFFECTS OF NUTRIENT OMISSION IN Caesalpinia echinata PLANTS}

\begin{abstract}
The objective of this study was to evaluate the growth, the morphological alterations and the mineral composition of brazilwood (Caesalpinia echinata) plants caused by mineral nutrients omission in a green house experiment. The experimental units were distributed in the green house according to a completely random design. The treatments, each repeated five times, were the following : check (natural soil), complete (N, P, K, Ca, $\mathrm{Mg}, \mathrm{S}, \mathrm{B}, \mathrm{Cu}, \mathrm{Mn}$, and $\mathrm{Zn}$ ) and a complete solution but for the omission of one of the nutrients in parenthesis. Each plot was represented by a plant growing in a $7 \mathrm{dm} 3$ vase filled with Quartzarenic Neosol. The analyzed variables were the following: visual nutritional deficiency symptoms, plant height, stem diameter, shoot dry matter, stem, branches and leaves included, and leaf nutrients level. The omission of nitrogen limited plant growth in height and shoot biomass production. The first visual deficiency symptoms were those due to $\mathrm{N}$ omission followed by those caused by $P, C a, M g, S, C u$, and Mn omissions. Later on the $K$ and $B$ deficiency symptoms became visible. The omission of a nutrient always caused its level in the leaves to be significantly lower than that found when it was not omitted.
\end{abstract}

Keywords : brazilwood, nutritional deficiency, diagnosis, forestal nutrition

\section{INTRODUÇÃO}

Caesalpinia echinata é uma das 140 espécies da subfamília Caesalpinoideae (LEWIS et al., 2005). Endêmica da faixa litorânea da Mata Atlântica, ocorre nos tabuleiros do Pliopleistoceno do Grupo Barreiras, desde o Rio Grande do Norte até o Rio de Janeiro (VELOSO et al., 1991).

A espécie se tornou rara em seu bioma de ocorrência natural (CONSERVATION INTERNATIONAL, 2011) devido à ação antrópica no período de colonização e extração ilegal impulsionada pela alta demanda de sua madeira no mercado internacional. A madeira de paubrasil é usada principalmente na manufatura de arcos dos instrumentos de corda (violino, viola, violoncelo e contrabaixo) em decorrência das propriedades vibracionais que são consideradas únicas (MATSUNAGA et al., 2000).

\footnotetext{
${ }^{1}$ Universidade Estadual Paulista - Jaboticabal, São Paulo, Brasil.
}

São encontrados poucos trabalhos de nutrição e adubação de pau-brasil. Aguiar e Pinho (1996) recomendam calagem com $500 \mathrm{~g}$ de calcário dolomítico e adubação com $500 \mathrm{~g}$ de farinha de osso e $500 \mathrm{~g}$ da fórmula 9-12-7 ( $\left.\mathrm{N}_{2} \mathrm{P}_{2} \mathrm{O}_{5}-\mathrm{K}_{2} \mathrm{O}\right)$ no plantio das mudas em campo. Contudo, outras pesquisas evidenciam que as aplicações de calcário dolomítico e cloreto de potássio em plantas de C. echinata resultam em menor crescimento em altura e diâmetro e número reduzido de folhas (AGUIAR et al., 1997).

A obtenção de informações sobre as exigências nutricionais do pau-brasil e do seu comportamento frente ao fornecimento limitado de nutrientes pode auxiliar no manejo de áreas de reflorestamento nos quais ele esteja incluído. Assim, o presente trabalho teve por objetivo avaliar o crescimento, as alterações morfológicas e os teores de nutrientes nas folhas de pau-brasil (Caesalpinia

Cerne, Lavras, v. 20, n. 1, p. 73-80, jan./mar. 2014 
echinata) decorrentes da omissão de nutrientes (N, P, K, Ca, $\mathrm{Mg}, \mathrm{S}, \mathrm{B}, \mathrm{Zn}, \mathrm{Mn}$ e $\mathrm{Cu}$ ) pela técnica do elemento faltante em experimento em casa de vegetação.

\section{MATERIAL E MÉTODOS}

O experimento foi conduzido em casa de vegetação, no Viveiro Experimental de Plantas Ornamentais e Florestais da Faculdade de Ciências Agrárias e Veterinárias (FCAV) da Universidade Estadual Paulista (Unesp), Câmpus de Jaboticabal - SP, em delineamento inteiramente casualizado com 12 tratamentos e cinco repetições. Os tratamentos foram: adubação completa (completo), sem adubação (testemunha) e adubação completa com omissão de nitrogênio, fósforo, potássio, cálcio, magnésio, enxofre, boro, cobre, manganês e zinco, um de cada vez, os quais foram designados respectivamente por: $-\mathrm{N},-\mathrm{P},-\mathrm{K},-\mathrm{Ca}$, -Mg, -S, -B, -Cu, -Mn e -Zn.

Foram usados vasos contendo $7 \mathrm{dm}^{3}$ de Neossolo Quartzarênico proveniente da camada de 0 a $20 \mathrm{~cm}$. O solo não havia recebido corretivos e fertilizantes nos sete anos anteriores à coleta.

A análise química do solo, segundo métodos descritos em Raij et al. (2001), apresentou os seguintes resultados: $\mathrm{P}$ resina, $8 \mathrm{mg} \mathrm{dm}^{-3}$; $\mathrm{MO}, 16 \mathrm{~g} \mathrm{dm}^{-3} ; \mathrm{pH}$ em $\mathrm{CaCl}_{2}, 3,8 ; \mathrm{K}^{+}, 0,3$ mmolc dm${ }^{-3} ; \mathrm{Ca}^{2+}, 1,0$ mmolc $\mathrm{dm}^{-3}$; $\mathrm{Mg}^{2+}, 0,5 \mathrm{mmol}_{\mathrm{c}} \mathrm{dm}^{-3} ; \mathrm{H}+\mathrm{Al}, 58 \mathrm{mmol}_{\mathrm{c}} \mathrm{dm}^{-3}$; CTC a $\mathrm{pH}$ 7, $60 \mathrm{mmol}_{\mathrm{c}} \mathrm{dm}^{-3}$ e saturação por bases (V), 3\%.

Ao solo dos tratamentos com adubação completa e de omissão de nutrientes (-N, -P, -K, -S, -B, -Cu, -Mn e -Zn) foram adicionados $\mathrm{CaCO}_{3}$ e $\mathrm{MgCO}_{3}$ (PRNT 100\%), para elevar a saturação por bases a $50 \%$, conforme recomendação de Gonçalves et al. (1997), empregando relação $\mathrm{Ca}: \mathrm{Mg}$ de 2:1. O tratamento testemunha não recebeu esses corretivos, o tratamento - $\mathrm{Ca}$ recebeu $\mathrm{MgCO}_{3}$ e o tratamento - $\mathrm{Mg}, \mathrm{CaCO}_{3}$. Foi usado pedaço de plástico para misturar os corretivos ao solo seco e, em seguida, ele foi recolocado no respectivo vaso para umedecimento com quantidade de água destilada suficiente para atingir $60 \%$ da capacidade de retenção. Nessas condições, o solo foi incubado por 30 dias. Após esse período foram aplicadas as soluções nutritivas. No tratamento completo, os nutrientes e as doses aplicadas, em $\mathrm{mg} \mathrm{dm}^{-3}$, foram: $85 \mathrm{de} \mathrm{N}, 75 \mathrm{de}$ P, 130 de K, 15 de S, 0,5 de B, 1 de Cu, 2 de Mn, 1,5 de Zn e 0,02 de Mo. Aos outros tratamentos foram aplicadas soluções com omissão isolada de cada um dos nutrientes em relação à solução completa, exceto Mo, que foi aplicado em todos, menos no tratamento-testemunha. As fontes dos nutrientes foram os sais p.a. apresentados na Tabela 1.

Tabela 1 - Adubos que foram empregados no preparo da solução de nutrientes, segundo o tratamento.

Table 1 - Fertilizers used to prepare the nutrients solution according to treatment.

\begin{tabular}{|c|c|}
\hline Tratamento & Fontes dos adubos \\
\hline Testemunha & - \\
\hline Completo & $\begin{array}{l}\mathrm{NH}_{4} \mathrm{H}_{2} \mathrm{PO}_{4} ; \mathrm{K}_{2} \mathrm{SO}_{4} ; \mathrm{KNO}_{3} ; \mathrm{CO}\left(\mathrm{NH}_{2}\right)_{2} ; \mathrm{H}_{3} \mathrm{BO}_{3} ; \mathrm{CuSO}_{4} \cdot 5 \mathrm{H}_{2} \mathrm{O} ; \\
\quad \mathrm{MnCl}_{2} \cdot 2 \mathrm{H}_{2} \mathrm{O} ; \mathrm{ZnSO}_{4} \cdot 7 \mathrm{H}_{2} \mathrm{O} ;\left(\mathrm{NH}_{4}\right)_{6} \mathrm{Mo}_{7} \mathrm{O}_{24} \cdot 4 \mathrm{H}_{2} \mathrm{O}\end{array}$ \\
\hline$-\mathrm{N}$ & $\begin{array}{c}\mathrm{KH}_{2} \mathrm{PO}_{4} ; \mathrm{K}_{2} \mathrm{SO}_{4} ; \mathrm{H}_{3} \mathrm{BO}_{3} ; \mathrm{CuSO}_{4} \cdot 5 \mathrm{H}_{2} \mathrm{O} ; \mathrm{MnCl}_{2} \cdot 2 \mathrm{H}_{2} \mathrm{O} ; \\
\mathrm{ZnSO}_{4} \cdot 7 \mathrm{H} 2 \mathrm{O} ;\left(\mathrm{NH}_{4}\right)_{6} \mathrm{Mo}_{7} \mathrm{O}_{24} \cdot 4 \mathrm{H}_{2} \mathrm{O}\end{array}$ \\
\hline$-\mathrm{P}$ & $\begin{array}{c}\mathrm{K}_{2} \mathrm{SO}_{4} ; \mathrm{KNO}_{3} ; \mathrm{CO}\left(\mathrm{NH}_{2}\right)_{2} ; \mathrm{H}_{3} \mathrm{BO}_{3} ; \mathrm{CuSO}_{4} \cdot 5 \mathrm{H}_{2} \mathrm{O} ; \mathrm{MnCl}_{2} .2 \\
\mathrm{H}_{2} \mathrm{O} ; \mathrm{ZnSO}_{4} \cdot 7 \mathrm{H}_{2} \mathrm{O} ;\left(\mathrm{NH}_{4}\right)_{6} \mathrm{Mo}_{7} \mathrm{O}_{24} \cdot 4 \mathrm{H}_{2} \mathrm{O}\end{array}$ \\
\hline$-K$ & $\begin{array}{l}\mathrm{NH}_{4} \mathrm{H}_{2} \mathrm{PO}_{4} ;\left(\mathrm{NH}_{4}\right)_{2} \mathrm{SO}_{4} ; \mathrm{CO}\left(\mathrm{NH}_{2}\right)_{2} ; \mathrm{H}_{3} \mathrm{BO}_{3} ; \mathrm{CuSO}_{4} \cdot 5 \mathrm{H}_{2} \mathrm{O} \\
\quad \mathrm{MnCl}_{2} \cdot 2 \mathrm{H}_{2} \mathrm{O} ; \mathrm{ZnSO}_{4} \cdot 7 \mathrm{H}_{2} \mathrm{O} ;\left(\mathrm{NH}_{4}\right)_{6} \mathrm{Mo}_{7} \mathrm{O}_{24} \cdot 4 \mathrm{H}_{2} \mathrm{O}\end{array}$ \\
\hline$-\mathrm{Ca}$ & $\begin{array}{l}\mathrm{NH}_{4} \mathrm{H}_{2} \mathrm{PO}_{4} ; \mathrm{K}_{2} \mathrm{SO}_{4} ; \mathrm{KNO}_{3}, \mathrm{CO}\left(\mathrm{NH}_{2}\right)_{2}, \mathrm{H}_{3} \mathrm{BO}_{3}, \mathrm{CuSO}_{4} \cdot 5 \mathrm{H}_{2} \mathrm{O} ; \\
\quad \mathrm{MnCl}_{2} \cdot 2 \mathrm{H}_{2} \mathrm{O} ; \mathrm{ZnSO}_{4} \cdot 7 \mathrm{H}_{2} \mathrm{O} ;\left(\mathrm{NH}_{4}\right)_{6} \mathrm{Mo}_{7} \mathrm{O}_{24} \cdot 4 \mathrm{H}_{2} \mathrm{O}\end{array}$ \\
\hline$-\mathrm{Mg}$ & $\begin{array}{l}\mathrm{NH}_{4} \mathrm{H}_{2} \mathrm{PO}_{4} ; \mathrm{K}_{2} \mathrm{SO}_{4} ; \mathrm{KNO}_{3} ; \mathrm{CO}\left(\mathrm{NH}_{2}\right)_{2} ; \mathrm{H}_{3} \mathrm{BO}_{3} ; \mathrm{CuSO}_{4} \cdot 5 \mathrm{H}_{2} \mathrm{O} ; \\
\quad \mathrm{MnCl}_{2} \cdot 2 \mathrm{H}_{2} \mathrm{O} ; \mathrm{ZnSO}_{4} \cdot 7 \mathrm{H}_{2} \mathrm{O} ;\left(\mathrm{NH}_{4}\right)_{6} \mathrm{Mo}_{7} \mathrm{O}_{24} \cdot 4 \mathrm{H}_{2} \mathrm{O}\end{array}$ \\
\hline$-S$ & $\begin{array}{c}\mathrm{NH}_{4} \mathrm{H}_{2} \mathrm{PO}_{4} ; \mathrm{KNO}_{3} ; \mathrm{CO}\left(\mathrm{NH}_{2}\right)_{2} ; \mathrm{H}_{3} \mathrm{BO}_{3} ; \mathrm{CuSO}_{4} \cdot 5 \mathrm{H}_{2} \mathrm{O} ; \mathrm{MnCl}_{2} . \\
2 \mathrm{H}_{2} \mathrm{O} ; \mathrm{ZnSO}_{4} \cdot 7 \mathrm{H}_{2} \mathrm{O} ;\left(\mathrm{NH}_{4}\right)_{6} \mathrm{Mo}_{7} \mathrm{O}_{24} \cdot 4 \mathrm{H}_{2} \mathrm{O}\end{array}$ \\
\hline$-B$ & $\begin{array}{c}\mathrm{NH}_{4} \mathrm{H}_{2} \mathrm{PO}_{4} ; \mathrm{K}_{2} \mathrm{SO}_{4} ; \mathrm{KNO}_{3} ; \mathrm{CO}\left(\mathrm{NH}_{2}\right)_{2} ; \mathrm{CuSO}_{4} \cdot 5 \mathrm{H}_{2} \mathrm{O} ; \mathrm{MnCl}_{2} . \\
2 \mathrm{H}_{2} \mathrm{O} ; \mathrm{ZnSO}_{4} \cdot 7 \mathrm{H}_{2} \mathrm{O} ;\left(\mathrm{NH}_{4}\right)_{6} \mathrm{Mo}_{7} \mathrm{O}_{24} \cdot 4 \mathrm{H}_{2} \mathrm{O}\end{array}$ \\
\hline$-\mathrm{Cu}$ & $\begin{array}{c}\mathrm{NH}_{4} \mathrm{H}_{2} \mathrm{PO}_{4} ; \mathrm{K}_{2} \mathrm{SO}_{4} ; \mathrm{KNO}_{3} ; \mathrm{CO}\left(\mathrm{NH}_{2}\right)_{2} ; \mathrm{H}_{3} \mathrm{BO}_{3} ; \mathrm{MnCl}_{2} .2 \mathrm{H}_{2} \mathrm{O} ; \\
\mathrm{ZnSO}_{4} \cdot 7 \mathrm{H}_{2} \mathrm{O} ;\left(\mathrm{NH}_{4}\right)_{6} \mathrm{Mo}_{7} \mathrm{O}_{24} \cdot 4 \mathrm{H}_{2} \mathrm{O}\end{array}$ \\
\hline$-\mathrm{Mn}$ & $\begin{array}{c}\mathrm{NH}_{4} \mathrm{H}_{2} \mathrm{PO}_{4} ; \mathrm{K}_{2} \mathrm{SO}_{4} ; \mathrm{KNO}_{3} ; \mathrm{CO}\left(\mathrm{NH}_{2}\right)_{2} ; \mathrm{H}_{3} \mathrm{BO}_{3} ; \mathrm{CuSO}_{4} \cdot 5 \mathrm{H}_{2} \mathrm{O} ; \\
\mathrm{ZnSO}_{4} \cdot 7 \mathrm{H}_{2} \mathrm{O} ;\left(\mathrm{NH}_{4}\right)_{6} \mathrm{Mo}_{7} \mathrm{O}_{24} \cdot 4 \mathrm{H}_{2} \mathrm{O}\end{array}$ \\
\hline$-Z n$ & $\begin{array}{c}\mathrm{NH}_{4} \mathrm{H}_{2} \mathrm{PO}_{4} ; \mathrm{K}_{2} \mathrm{SO}_{4} ; \mathrm{KNO}_{3} ; \mathrm{CO}\left(\mathrm{NH}_{2}\right)_{2} ; \mathrm{H}_{3} \mathrm{BO}_{3} ; \mathrm{MnCl}_{2} \cdot 2 \mathrm{H}_{2} \mathrm{O} ; \\
\mathrm{CuSO}_{4} \cdot 5 \mathrm{H}_{2} \mathrm{O} ;\left(\mathrm{NH}_{4}\right)_{6} \mathrm{Mo}_{7} \mathrm{O}_{24} \cdot 4 \mathrm{H}_{2} \mathrm{O}\end{array}$ \\
\hline
\end{tabular}

Sete dias após a aplicação dos nutrientes, foi transplantada uma muda de pau-brasil por vaso, proveniente de sacos de plástico contendo $650 \mathrm{~cm}^{3}$ de substrato a base de subsolo (Latossolo Vermelho Distroférrico) e esterco bovino, na proporção volumétrica de 3:1 e com dois anos de idade, altura média de $26 \mathrm{~cm}$ e diâmetro do caule de $5,5 \mathrm{~mm}$, medido a $3 \mathrm{~cm}$ do solo. No transplantio, as mudas foram lavadas em água corrente para retirada do substrato.

A umidade do solo foi mantida a $60 \%$ da capacidade máxima de retenção de água, durante todo o período de condução do experimento, com água destilada. A umidade foi monitorada por meio de pesagens periódicas em balança digital, marca Micheletti, com capacidade de $15 \mathrm{~kg}$ e precisão de $5 \mathrm{~g}$.

Foi realizada adubação de cobertura aos 230 e 314 dias após o transplantio, com a aplicação, em mg dm-3, das seguintes doses de nutrientes: 45 de N, 45 de K e 18,4 de $\mathrm{S}$, exceto nos tratamentos $-\mathrm{N},-\mathrm{K}$ e $-\mathrm{S}$, respectivamente, e também no tratamento testemunha. As fontes usadas foram:

Cerne, Lavras, v. 20, n. 1, p. 73-80, jan./mar. 2014 
sulfato de potássio no tratamento -N; sulfato de amônio mais ureia no tratamento $-\mathrm{K}$; e nitrato de potássio mais ureia no tratamento -S..

Foram realizadas avaliações dos efeitos dos tratamentos na altura das plantas aos 90, 150,240,330 e 360 dias após o transplantio. O experimento foi conduzido durante um ano, pois trata-se de espécie de crescimento lento, e esse período foi suficiente para que as plantas respondessem aos tratamentos e manifestassem os sintomas de deficiência de nutrientes, sem que houvesse comprometimento do desenvolvimento vegetativo das plantas em função do volume de solo de cada vaso.

Aos 360 dias, foi feita a medida do diâmetro do colo das plantas, com paquímetro, as plantas foram cortadas rente ao solo e secas em estufa com circulação forçada de ar a $65-70{ }^{\circ} \mathrm{C}$ até massa constante. Em seguida, foram determinados a massa de matéria seca da parte aérea e os teores de nitrogênio, fósforo, potássio, cálcio, magnésio, enxofre, cobre, manganês e zinco nas folhas, de acordo com Carmo et al. (2000).

Foram realizadas as análises de variância dos dados obtidos e as médias foram comparadas pelo teste de Tukey a $5 \%$ de probabilidade.

\section{RESULTADOS E DISCUSSÃO}

\subsection{Sintomas visuais de deficiência de macro e micronutrientes}

Os sintomas de deficiência de nitrogênio foram os primeiros a se manifestar, com início aos 3 meses a partir do transplantio e agravamento nos 9 meses subsequentes. Foi observado raquitismo das plantas, amarelecimento generalizado das folhas, com clorose mais intensa nas folhas mais velhas, devido à alta mobilidade desse nutriente para as regiões em crescimento (MALAVOLTA et al., 1997). Os sintomas de deficiência do tratamento -P foram observados primeiramente nas folhas novas, a partir de 3 meses após o transplantio. Até 12 meses após $\mathrm{o}$ transplantio, os sintomas foram se definindo em clorose seguida de arroxeamento, necrose pontual nos espaços internervais do limbo e curvamento marginal.

No tratamento com omissão de potássio foi observado, a partir de 8 meses do transplantio, clorose internerval, necrose nas margens e enrugamento das folhas mais velhas. Na deficiência de potássio há acúmulo de ácido abscísico, o qual ativa a NADPH-oxidase e a formação de radicais livres dependentes de NADPH-oxidase (JIANG;
ZHANG, 2002), que causam degradação do pigmento clorofiliano e danos às membranas (CAKMAK, 2005), levando a necrose dos tecidos.

A carência de cálcio provocou anormalidades no crescimento das plantas a partir de 4 meses após o transplantio. O cálcio é importante na manutenção da membrana plasmática e da rigidez da parede celular (MATHUIS, 2009), o que pode explicar as deformações observadas, como o encarquilhamento nos órgãos vegetativos em crescimento. Verificou-se também clorose, e com a evolução da deficiência, aos 12 meses após o transplantio, as plantas começaram perder as folhas e houve a morte da gema apical.

A deficiência de magnésio caracterizou-se pela clorose nas folhas novas e recém-maduras, a partir de 4 meses após o transplantio. Com o agravamento do sintoma, algumas folhas apresentaram pequenos pontos necróticos de cor castanha e abscisão foliar no final do período de condução do experimento. É provável que tenha ocorrido acúmulo de fotossintatos devido à inibição do carregamento no floem,a induzida pela deficiência de magnésio, o que causou desorganização e necrose do floema (FINK, 1991).

As plantas que não receberam enxofre exibiram clorose nas folhas mais novas a partir de 4 meses após o transplantio e redução no crescimento em comparação ao tratamento completo, sintomas similares aos verificados em plantas de Tectona grandis (BARROSO et al., 2005). No período de 5 a 12 meses de idade, esses sintomas foram se intensificando.

Início de envassouramento, resultante da perda de dominância apical, e deformação nas folhas novas, foram observados com a omissão de boro, a partir de 9 meses de idade, como relatado por Mendonça et al. (1999) em plantas de Myracrodruon urundeuva. O boro participa no transporte de carboidratos e na formação da parede celular.Assim, sua deficiência resulta em parede celular desestruturada, menos lignificada e no comprometimento da integridade da membrana celular (MALAVOLTA, 2006).

Para os demais micronutrientes, $\mathrm{Cu}, \mathrm{Mn}$ e $\mathrm{Zn}$, a definição dos sintomas de deficiência iniciou a aproximadamente 4 meses após o transplantio. As plantas do tratamento com omissão de cobre apresentaram raquitismo, tortuosidades, clorose e necrose nas folhas novas, além de nervuras salientes. Alguns desses sintomas de deficiência também foram relatados para as espécies Bertholletia excelsa (CAMARGO; MURAOKA, 2007) e Myracrodruon urundeuva (MENDONÇA et al., 1999).

Cerne, Lavras, v. 20, n. 1, p. 73-80, jan./mar. 2014 
A omissão de manganês provocou aparecimento de sintomas caracterizados por clorose das folhas mais novas, com reticulado grosso das nervuras verdes sobre fundo amarelado, devido à distribuição irregular de clorofila.Em plantas de Bertholletia excelsa foram descritos sintomas semelhantes (CAMARGO; MURAOKA, 2007).

Os sintomas observados com a omissão de zinco em plantas de C. echinata incluíram superbrotação, folhas cloróticas, área foliar reduzida, necrose de folhas velhas e nas margens de folhas novas. Esse elemento está envolvido no metabolismo de auxina.Assim, sua carência pode resultar em perda da dominância apical, resultando em intensa brotação lateral (EPSTEIN, 1995). O zinco participa também da divisão celular e da síntese proteica (MATHUIS, 2009), de modo que na omissão desse nutriente, tais processos devem ter sido prejudicados, o que pode explicar o crescimento reduzido das folhas.

\subsection{Altura e Diâmetro}

A altura das mudas aos 90 dias após o transplantio foi de $48,05 \mathrm{~cm}$, sem diferença entre os tratamentos. Também não houve diferença no crescimento em altura entre as plantas do tratamento testemunha e as dos demais tratamentos durante a condução do experimento (Tabela 2).

O nitrogênio foi o nutriente mais limitante ao crescimento das mudas. Nas quatro avaliações, a altura das plantas do tratamento $-\mathrm{N}$ foi menor do que do tratamento com adubação completa. Entretanto, Aguiar et al. (1997) verificaram efeito negativo da aplicação de nitrogênio no crescimento em altura das mudas de $C$. echinata em casa de vegetação. A diferença dos resultados entre os dois experimentos, provavelmente, se deve aos atributos químicos e físicos distintos dos solos usados e da idade das mudas usadas por Aguiar et al. (1997) ser maior do que as do presente trabalho.

Aos 150 dias após o transplantio, as plantas do tratamento - $\mathrm{Ca}$ apresentaram maior altura do que as do tratamento - $\mathrm{Ne}$-S. As mudas continuaram a se desenvolver mais em altura no tratamento com omissão de cálcio do que na carência de nitrogênio até o encerramento do experimento. Aguiar et al. (1997) verificaram menor altura e menor diâmetro em plantas de pau-brasil, aos 39 meses de idade, com a aplicação de calcário. C. echinata deve ser pouco exigente em cálcio, pois a espécie é endêmica da Mata Atlântica, onde os solos geralmente possuem baixo teor de cálcio (BARRETO et al., 2006).

Cerne, Lavras, v. 20, n. 1, p. 73-80, jan./mar. 2014
O maior crescimento em diâmetro de colo ocorreu nas plantas do tratamento completo, não diferindo dos outros tratamentos. Corroborando com os resultados do presente trabalho, em plantas de Tabebuia impetiginosa, os diâmetros observados nos tratamentos $-\mathrm{K},-\mathrm{Ca},-\mathrm{Mg},-\mathrm{B}$ e $-\mathrm{Zn}$ foram similares estatisticamente ao do tratamento com adubação completa (SOUZA et al., 2006).

Tabela 2 - Médias de altura e de diâmetro de colo de plantas de Caesalpinia echinata, em função dos tratamentos, e resultados das análises estatísticas.

Table 2 - Height and stem diameter of Caesalpinia echinata plants as influenced by fertilizers and results of the statistical analyses.

\begin{tabular}{ccccccc}
\hline \multirow{2}{*}{ Tratamentos } & \multicolumn{5}{c}{ Média das Alturas (cm) } & $\begin{array}{c}\text { Diâmetro } \\
(\mathrm{mm})\end{array}$ \\
\cline { 2 - 7 } & 90 dias & 150 dias & 240 dias & 330 dias & 360 dias & 360 dias \\
\hline Completo & 48,33 & $71,33 \mathrm{ab}$ & $96,33 \mathrm{a}$ & $109,33 \mathrm{a}$ & $109,67 \mathrm{a}$ & $10,9 \mathrm{abc}$ \\
Testemunha & 41,60 & $53,40 \mathrm{ab}$ & $66,40 \mathrm{ab}$ & $71,80 \mathrm{ab}$ & $78,00 \mathrm{ab}$ & $8,4 \mathrm{c}$ \\
-N & 43,70 & $46,20 \mathrm{~b}$ & $56,60 \mathrm{~b}$ & $65,60 \mathrm{~b}$ & $64,00 \mathrm{~b}$ & $9,0 \mathrm{bc}$ \\
-P & 49,00 & $58,33 \mathrm{ab}$ & $78,33 \mathrm{ab}$ & $87,67 \mathrm{ab}$ & $91,33 \mathrm{ab}$ & $10,1 \mathrm{abc}$ \\
-K & 52,40 & $66,40 \mathrm{ab}$ & $79,00 \mathrm{ab}$ & $91,40 \mathrm{ab}$ & $93,20 \mathrm{ab}$ & $10,3 \mathrm{abc}$ \\
-Ca & 54,90 & $79,80 \mathrm{a}$ & $97,00 \mathrm{a}$ & $112,20 \mathrm{a}$ & $110,20 \mathrm{a}$ & $11,4 \mathrm{ab}$ \\
-Mg & 51,80 & $68,20 \mathrm{ab}$ & $78,40 \mathrm{ab}$ & $82,40 \mathrm{ab}$ & $83,40 \mathrm{ab}$ & $12,5 \mathrm{a}$ \\
-S & 43,60 & $50,30 \mathrm{~b}$ & $81,40 \mathrm{ab}$ & $104,20 \mathrm{ab}$ & $104,60 \mathrm{ab}$ & $10,3 \mathrm{abc}$ \\
-B & 40,40 & $54,20 \mathrm{ab}$ & $75,20 \mathrm{ab}$ & $86,80 \mathrm{ab}$ & $85,40 \mathrm{ab}$ & $10,2 \mathrm{abc}$ \\
-Cu & 46,67 & $63,00 \mathrm{ab}$ & $84,67 \mathrm{ab}$ & $97,67 \mathrm{ab}$ & $98,67 \mathrm{ab}$ & $10,7 \mathrm{abc}$ \\
-Mn & 53,50 & $67,00 \mathrm{ab}$ & $93,75 \mathrm{a}$ & $98,75 \mathrm{ab}$ & $99,00 \mathrm{ab}$ & $11,4 \mathrm{ab}$ \\
$-\mathrm{Zn}$ & 50,67 & $62,00 \mathrm{ab}$ & $70,67 \mathrm{ab}$ & $83,67 \mathrm{ab}$ & $85,67 \mathrm{ab}$ & $10,3 \mathrm{abc}$ \\
\hline Teste F & $1,04^{\mathrm{ns}}$ & $3,72 * *$ & $3,27 * *$ & $3,05 * *$ & $3,00 * *$ & $4,2 * *$ \\
CV (\%) & 21,72 & 17,77 & 17,76 & 19,30 & 18,43 & 11,23 \\
\hline
\end{tabular}

Médias seguidas de mesma letra não diferem entre si pelo teste de Tukey $(\mathrm{P}>0,05) .{ }^{\mathrm{n} s}=$ não significativo $(\mathrm{P}>0,05) ;{ }^{*} \mathrm{e}^{* *}=$ significativos $(\mathrm{P}<0,05)$ e $(\mathrm{P}<0,01)$, respectivamente.

A omissão de cobre não afetou o crescimento em altura e não diferiu do tratamento completo em relação ao crescimento em diâmetro. Resultados semelhantes foram relatados por Mendonça et al. (1999) em plantas de Myracrodruon urundeuva.

O crescimento em diâmetro das plantas nos tratamentos - $\mathrm{Ca},-\mathrm{Mg}$ e -Mn foi maior do que o observado na testemunha. $\mathrm{O}$ incremento em diâmetro se dá pelo crescimento secundário, em que a deposição de lignina é requisito. A carência de magnésio altera pouco esse processo, mas a deficiência de cálcio afeta a lignificação, bem como as de manganês, boro e cobre (MARSCHNER, 1995; SILVEIRA; HIGASHI, 2003). Assim, os resultados positivos observados na carência de cálcio, bem como na de manganês, em comparação à testemunha, se deve 
possivelmente ao fornecimento dos outros nutrientes necessários ao crescimento secundário.

O diâmetro das plantas deficientes em nitrogênio diferiu apenas do tratamento $-\mathrm{Mg}$, que como foi comentado anteriormente, tem pouca participação no incremento em diâmetro, diferente do nitrogênio que é necessário para a formação e diferenciação de tecidos (SILVEIRA; HIGASHI, 2003; MATHUIS, 2009).

\subsection{Produção de matéria seca da parte aérea e teores de nutrientes}

A produção de matéria seca das plantas do tratamento completo foi maior do que a observada na testemunha e na omissão de nitrogênio, não diferindo dos demais tratamentos aos 360 dias após o transplantio (Tabela 3). A omissão de nitrogênio também reduziu a produção de biomassa na parte aérea de mudas de Annona muricata (BATISTA et al., 2003) e Tabebuia impetiginosa (SOUZA et al., 2006).

A produção de biomassa foi menor no tratamento testemunha do que nos tratamentos completo, -Ca e -Mn.

$\mathrm{Na}$ omissão de cálcio observou-se redução do teor de fósforo e de manganês nas folhas e maior produção de biomassa do que a testemunha. Essa maior produção de biomassa pode estar relacionada à diminuição do teor de manganês nas folhas induzida pelo tratamento -Ca. A aplicação de cálcio tende a elevar o $\mathrm{pH}$ do meio e diminuir a disponibilidade desses nutrientes (MALAVOLTA, 2006). Apesar da análise de variância pelo teste $F$ não ter acusado efeito dos tratamentos no teor de nitrogênio nas folhas, ele foi duas vezes maior na omissão de cobre quando comparado com o tratamento -P. A deficiência de fósforo afeta a síntese proteica, o que pode ter resultado em acúmulo de aminoácidos nas raízes, provocando a inibição da atividade de transportadores de nitrato na membrana

Tabela 3 - Médias de massa de matéria seca da parte aérea e teor de nutrientes nas folhas de plantas de Caesalpinia echinata aos 360 dias após o transplantio em função dos tratamentos e resultados das análises estatísticas.

Table 3 - Shoot dry mater mass and nutrient leaf contents averages of Caesalpinia echinata plants at 360 days after transplantation in function of treatments and results of statistical analysis.

\begin{tabular}{|c|c|c|c|c|c|c|c|c|c|}
\hline \multirow{3}{*}{ Tratamentos } & \multirow{3}{*}{$\begin{array}{c}\begin{array}{c}\text { Matéria } \\
\text { seca }\end{array} \\
\text { g planta }^{-1} \\
\text { Parte aérea }\end{array}$} & \multicolumn{8}{|c|}{ Teor nas folhas } \\
\hline & & \multicolumn{6}{|c|}{ 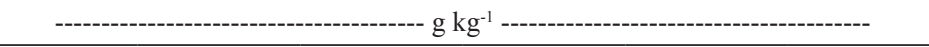 } & \multicolumn{2}{|c|}{-------- $\mathrm{mg} \mathrm{kg}^{-1}$-------- } \\
\hline & & $\mathrm{N}$ & $\mathrm{P}$ & $\mathrm{K}$ & $\mathrm{Ca}$ & $\mathrm{Mg}$ & $\mathrm{S}$ & $\mathrm{Mn}$ & $\mathrm{Zn}$ \\
\hline Completo & 76,66 a & $14,84 \mathrm{ab}$ & $3,42 \mathrm{~cd}$ & 9,74 abcde & $8,84 \mathrm{abc}$ & 1,38 cde & 4,34 cde & 25,20 cde & $14,60 \mathrm{~b}$ \\
\hline Testemunha & $30,16 \mathrm{~b}$ & $11,21 \mathrm{ab}$ & $6,20 \mathrm{a}$ & $6,65 \mathrm{de}$ & $6,38 \mathrm{bc}$ & 1,88 abcd & $3,15 \mathrm{e}$ & $160,25 \mathrm{a}$ & $29,50 \mathrm{a}$ \\
\hline$-\mathrm{N}$ & $25,61 \mathrm{~b}$ & $11,20 \mathrm{ab}$ & $5,03 \mathrm{ab}$ & $12,14 \mathrm{abc}$ & $12,84 \mathrm{a}$ & 1,50 bcde & $5,27 \mathrm{bcd}$ & 38,00 bcde & $23,40 \mathrm{ab}$ \\
\hline$-\mathrm{P}$ & $44,56 \mathrm{ab}$ & $9,95 \mathrm{~b}$ & $2,72 \mathrm{~d}$ & 8,20 cde & $7,90 \mathrm{abc}$ & 1,70 abcd & 4,02 cde & $19,75 \mathrm{e}$ & $16,00 \mathrm{~b}$ \\
\hline$-K$ & $59,41 \mathrm{ab}$ & $11,98 \mathrm{ab}$ & $3,92 \mathrm{bcd}$ & $5,34 \mathrm{e}$ & $10,96 \mathrm{ab}$ & $2,46 \mathrm{a}$ & 3,38 ed & $38,60 \mathrm{bcd}$ & $20,60 \mathrm{ab}$ \\
\hline$-\mathrm{Ca}$ & $78,85 \mathrm{a}$ & $11,29 \mathrm{ab}$ & $3,17 \mathrm{~cd}$ & 9,18 bcde & $4,14 \mathrm{c}$ & $2,22 \mathrm{ab}$ & $3,10 \mathrm{e}$ & $48,60 \mathrm{~b}$ & $15,40 \mathrm{~b}$ \\
\hline$-\mathrm{Mg}$ & $48,47 \mathrm{ab}$ & $11,73 \mathrm{ab}$ & $4,24 \mathrm{bc}$ & 10,10 abcd & $8,84 \mathrm{abc}$ & $0,80 \mathrm{e}$ & $7,00 \mathrm{ab}$ & $40,60 \mathrm{bc}$ & $16,00 \mathrm{~b}$ \\
\hline$-S$ & $55,89 \mathrm{ab}$ & $11,71 \mathrm{ab}$ & $3,44 \mathrm{~cd}$ & 9,72 abcde & $6,46 \mathrm{~cd}$ & $1,22 \mathrm{de}$ & $0,80 \mathrm{f}$ & $20,80 \mathrm{de}$ & $13,20 \mathrm{~b}$ \\
\hline$-B$ & $56,78 \mathrm{ab}$ & $18,68 \mathrm{ab}$ & $4,17 \mathrm{bc}$ & $12,83 \mathrm{ab}$ & $10,35 \mathrm{ab}$ & $2,15 \mathrm{abc}$ & 4,71 cde & 22,50 cde & $18,00 \mathrm{~b}$ \\
\hline$-\mathrm{Cu}$ & $48,73 \mathrm{ab}$ & $20,65 \mathrm{a}$ & $3,85 \mathrm{bcd}$ & $14,10 \mathrm{a}$ & $9,90 \mathrm{ab}$ & 1,82 abcd & $7,54 \mathrm{a}$ & $20,20 \mathrm{de}$ & ------- \\
\hline$-\mathrm{Mn}$ & $80,76 \mathrm{a}$ & $11,00 \mathrm{ab}$ & $3,24 \mathrm{~cd}$ & $11,80 \mathrm{abc}$ & $9,78 \mathrm{ab}$ & 1,76 abcd & $5,72 \mathrm{abc}$ & 27,20 cde & $15,60 \mathrm{~b}$ \\
\hline$-\mathrm{Zn}$ & $51,52 \mathrm{ab}$ & $11,38 \mathrm{ab}$ & $3,71 \mathrm{bcd}$ & $12,02 \mathrm{abc}$ & $9,04 \mathrm{abc}$ & 1,54 bcde & 4,47 cde & 22,20 cde & $15,40 \mathrm{~b}$ \\
\hline Teste F & $4,84 * *$ & 2,82 & $9,07 * *$ & $7,72 * *$ & $8,46 * *$ & $8,46 * *$ & $18,93 * *$ & $88,47 * *$ & $4,68 * *$ \\
\hline $\mathrm{CV}(\%)$ & 31,07 & 33,23 & 16,48 & 19,73 & 20,61 & 20,61 & 20,80 & 21,55 & 26,54 \\
\hline
\end{tabular}

Médias seguidas de mesma letra não diferem entre si pelo teste de Tukey $(\mathrm{P}>0,05) .{ }^{* *}=$ significativos $(\mathrm{P}<0,01)$

Cerne, Lavras, v. 20, n. 1, p. 73-80, jan./mar. 2014 
(IMSANDE; TOURAINE, 1994) e, consequentemente, reduzindo a absorção de nitrogênio.

Com a omissão de nitrogênio, observou-se maior teor de cálcio nas folhas do que o verificado nos demais tratamentos. De acordo com Malavolta (2006), a absorção de $\mathrm{Ca}^{2+}$ pode ser diminuída mediante a alta concentração de $\mathrm{K}^{+}, \mathrm{Mg}^{2+}$ e $\mathrm{NH}^{4+}$ no meio.

Houve aumento do teor de magnésio nas folhas com a omissão de potássio em comparação com o tratamento completo. Porém, Keil et al. (2009) verificaram aumento do teor de magnésio no tratamento -K em plantas de Ocotea odorifera.

Os teores de fósforo, magnésio, enxofre, manganês e zinco foram estatisticamente menores nos tratamentos em que houve respectivamente omissão desses elementos, quando comparados à testemunha. Contudo, no tratamento $-\mathrm{N}$, os teores de fósforo e zinco foram semelhantes estatisticamente à testemunha.

O teor de enxofre nas folhas foi aproximadamente cinco vezes menor quando o elemento foi omitido, em comparação ao tratamento completo, mas essa redução acentuada não resultou em menor produção de biomassa. Os outros tratamentos não diferiram do tratamento completo em relação aos teores de nutrientes nas folhas.

A omissão de manganês resultou em maior produção de matéria seca que a testemunha. Em todos os tratamentos houve aplicação de carbonatos, com exceção da testemunha, o que elevou o $\mathrm{pH}$ e consequentemente diminuiu a disponibilidade desse micronutriente no solo. Como resultado houve menor absorção e teor de manganês nas folhas, mesmo nos tratamentos em que ele foi aplicado. Os resultados observados permitem sugerir que a concentração de manganês no solo natural usado (testemunha) é superior ao adequado para o pau-brasil, causando toxicidade as plantas da espécie.

A omissão de cobre afetou positivamente o teor de enxofre nas folhas em comparação aos tratamentos completo e testemunha,

Não houve efeitos significativos dos tratamentos nos teores de cobre e ferro nas folhas, e o teores médios obtidos foram 2,17 mg kg-1 de Cu e 118,38 $\mathrm{mg} \mathrm{kg}^{-1}$ de Fe.

O teor de zinco nas folhas das plantas do tratamentotestemunha foi maior do que o das plantas que receberam adubação completa, o que pode ser em parte devido à maior acidez do solo no tratamento testemunha. A disponibilidade de zinco para as plantas, do mesmo modo como ocorre com o manganês, diminui com o aumento do valor de $\mathrm{pH}$ (Havlin et al., 2005).

\section{CONCLUSÕES}

A omissão de nitrogênio em Neossolo Quartzarênico limitou o crescimento em altura das plantas de pau-brasil e as omissões dos demais nutrientes não interferiram no crescimento das plantas.

A ordem cronológica de manifestação de sintomas visuais de deficiência foi $\mathrm{N}, \mathrm{P}, \mathrm{Ca}, \mathrm{Mg}, \mathrm{S}, \mathrm{Cu}, \mathrm{Mn}, \mathrm{K}$ e B, iniciando aos 3 meses do transplantio.

A matéria seca da parte aéreas das plantas cultivadas no solo natural, sem calagem e adubação, foi $60 \%$ menor do que no tratamento completo. A omissão de $\mathrm{N}$ limitou a produção de matéria seca da parte aérea em relação ao tratamento completo. Para cada nutriente, houve diferença entre as médias de teores nas folhas em função dos tratamentos. Os teores de $\mathrm{P}$, foram maiores no tratamento testemunha do que no tratamento completo pelo efeito de diluição, e os teores de Mn e Zn também foram maiores no tratamento-testemunha por causa da maior acidez no solo.

\section{REFERÊNCIAS}

AGUIAR, F. F.; PINHO, R. A. Pau-brasil: Caesalpinia echinata Lam. 2. ed. São Paulo: Instituto de Botânica, 1996. 14 p. (Folheto, 18).

AGUIAR, F. F. A. et al. Influência da adubação no crescimento de mudas de Pau-Brasil (Caesalpinia echinata Lam.). Revista Brasileira de Horticultura Ornamental, Campinas, v. 3, n. 2, p. 42-49, 1997.

BARRETO, A. C. et al. Características químicas e físicas de um solo sob floresta, sistema agroflorestal e pastagem no sul da Bahia. Caatinga, Mossoró, v. 19, n. 4, p.415-425, 2006.

BARROSO, D. G. et al. Diagnóstico de deficiências de macronutrientes em mudas de teca. Revista Árvore, Viçosa, v. 29, n. 5, p.671-679, 2005.

BATISTA, M. M. F. et al. Efeito da omissão de macronutrientes no crescimento, nos sintomas de deficiências nutricionais e na composição mineral em gravioleiras (Annona muricata). Revista Brasileira de Fruticultura, Jaboticabal, v. 25, n. 2, p. 315-318, 2003.

Cerne, Lavras, v. 20, n. 1, p. 73-80, jan./mar. 2014 
CAKMAK, I. The role of potassium in alleviating detrimental effects of abiotic stresses in plants. Journal of Plant Nutrition and Soil Science, Oldenburg, v. 168, n. 4, p. 521-530, 2005.

CAMARGO, S. L.; MURAOKA, T. Teores, acúmulo e redistribuição de micronutrientes em castanheira do Brasil. Revista Agricultura Tropical, Cuiabá, v. 9, p. 144-154, 2007.

CARMO, C. A. F. S. et al. Métodos de análise de tecidos vegetais utilizados na Embrapa Solos. Rio de Janeiro: Embrapa Solos, 2000. p.41. (Circular técnica, 6)

CONSERVATION INTERNATIONAL. Biodiversity Hotspot: Atlantic Forest - Unique and threatened biodiversity. Disponível em: < http://www. biodiversityhotspots.org/xp/hotspots/atlantic_forest/ Pages/biodiversity.aspx $>$. Acesso em: 7 jul. 2011.

EPSTEIN, E. Plants and inorganic nutrients. In: HOPKINS, W.G. Introduction to plant physiology. Toronto: Wiley \& Sons, 1995. p. 65-80.

FINK, S. Structural changes in conifer needles due to $\mathrm{Mg}$ and $\mathrm{K}$ deficiency. Fertilizer Research, Dordrecht, v. 27, p. 23-7. 1991.

GONÇALVES, J. L. M.; RAIJ, B.; GONÇALVES, J. C. Florestais. In: RAIJ, B. Recomendações de adubação e calagem para o Estado de São Paulo. Campinas: Instituto Agronômico, 1997. p. 247-259.

HAVLIN, J.L.; BEATON, J.D.; TISDALE, S.L.; NELSON, W.L. Soil fertility and fertilizers. An introduction to nutrient management. 7. ed. New Jersey: Pearson Education, 2005. 515p.

IMSANDE, J.; TOURAINE, B. N. Demand and regulation of nitrate uptake. Plant Physiology, Lancaster, v. 105, p. 3-7, 1994.

JIANG, M. Y.; ZHANG, J. H. Involvement of plasmamembrane NADPH oxidase in abscisic acid- and water stress-induced antioxidant defense in leaves of maize seedlings. Planta, Berlin, v. 215, p. 1022-1030, 2002.

KEIL, S. S.; REISSMANN, C. B.; PELLICO NETTO, S. Crescimento e nutrição de Ocotea odorifera (Canela Sassafrás) submetido à fertilização e à omissão de nutrientes. Pesquisa Florestal Brasileira, Colombo, n.58, p.17-27, 2009.
LEWIS, G. et al. (Eds.). Legumes of the World.

Richmond: Royal Botanic Gardens, Kew. 2005. 592 p.

MALAVOLTA, E.; VITTI, G. C.; OLIVEIRA, S. A. Avaliação do estado nutricional das plantas: princípios e aplicações. Piracicaba: Associação Brasileira para a Pesquisa da Potassa e do Fosfato, 1997. $319 \mathrm{p}$.

MALAVOLTA, E. Manual de adubação de plantas. São Paulo: Agronômica Ceres, 2006. 638 p

MARSCHNER, H. Mineral nutrition of higher plants. 2 Ed. New York: Academic Press, 1995. 889 p.

MATSUNAGA, M. et al. Vibrational property changes of spruce wood by impregnation with water-soluble extractives of Pernambuco (Guilandina echinata Spreng) II: structural analysis of extractive components. Journal of Wood Science, Tokyo, v. 46, n. 1, p. $257-$ $353,2000$.

MENDONÇA, A. V. R. et al. Exigências nutricionais de Myracrodruon urundeuva Fr. All. (Aroeira-do-sertão).

Cerne, Lavras, v. 5, n. 2, p 65-75, 1999.

RAIJ, B. van; ANDRADE, J. C.; CANTARELLA, H.; QUAGGIO, J. A., eds. Análise química para avaliação da fertilidade de solos tropicais. Campinas: Instituto Agronômico, 2001. 285p.

SILVEIRA, R. L. V. A.; HIGASHI, E. N. Aspectos nutricionais envolvidos na ocorrência de doenças com ênfase para o eucalipto. Piracicaba, IPEF, p. 1-13. 2003. (Circular técnica, 200).

SOUZA, P. A.; VENTURIN, N.; MACEDO, R. L. G. Adubação mineral do ipê-roxo (Tabebuia impetiginosa). Ciência Florestal, Santa Maria, v. 16, n. 3, p. 261-70, 2006.

Recebido: 1 de novembro de 2011; aceito: 22 de julho de 2013.

Cerne, Lavras, v. 20, n. 1, p. 73-80, jan./mar. 2014 
